\title{
Etiología de las vasculitis cutáneas: utilidad de una aproximación sistémica
}

\author{
Caroline Chanussot-Deprez¹, María Elisa Vega-Memije², Luis Flores-Suárez³, Celia Ríos-Romero4, \\ Javier Cabiedes-Contreras ${ }^{3 \dagger}$, Edgardo Reyes ${ }^{5}$ y Lucia Rangel-Gamboa ${ }^{2}$ \\ ${ }^{1}$ Servicio de Dermatología Hospital General-PEMEX, Veracruz, Ver., México; ${ }^{2}$ Subdirección de Investigación Biomédica, Hospital General Dr. Manuel \\ Gea González, Ciudad de México, México; ${ }^{3}$ Departamento de Inmunología y Reumatología, Instituto Nacional de Ciencias Médicas y Nutrición, \\ Ciudad de México, México; ${ }^{4}$ Práctica privada, Querétaro, México; ${ }^{5}$ Departamento de Patología, Instituto Nacional de Ciencias Médicas y Nutrición, \\ Ciudad de México, México
}

\section{Resumen}

Las vasculitis cutáneas (VC), primarias o como manifestación de enfermedades sistémicas, constituyen un reto diagnóstico. Objetivo: Determinar las causas de VC. Métodos: Se incluyeron pacientes con diagnóstico de CV, a los cuales se les realizó valoración clínica, biopsia cutánea y exámenes de laboratorio. En la mayoría de los casos se realizó inmunofluorescencia directa. Los casos se clasificaron con los criterios del American College of Rheumatology (ACR) y la Chapel Hill Consensus Conference (CHCC). Resultados: Se incluyeron 32 pacientes; la frecuencia fue mayor en mujeres (71.8\%). Los niños presentaron VC asociadas a medicamentos o púrpura de Schönlein-Henoch (PSH). En adultos se reportó con más frecuencia $P S H$, vasculitis asociada a lupus eritematoso sistémico y vasculitis paraneoplásicas; otros diagnósticos etiológicos incluyeron poliarteritis nodosa (PAN), poliangeítis microscópica (PAM), vasculitis trombótica (pospuerperal), síndrome antifosfolípidos (SAF), síndrome de Churg-Strauss (SCS) y VC asociada a medicamentos. Utilizando los criterios del ACR y la CHCC para vasculitis se clasificó el 50\% de los casos. Discusión: En el Hospital Gea, durante este trabajo, el diagnóstico etiológico de las CV se incrementó más del doble. Sin embargo, en relación a los diagnósticos vasculitis por hipersensibilidad (VHS) y PSH ninguna de las clasificaciones utilizadas contaba con criterios específicos. Seis pacientes permanecieron sin clasificar. Observamos que los estudios de crioglobulinas y serología para hepatitis no son útiles como estudios iniciales, salvo que la historia clínica del paciente lo sugiera. Los pacientes sin clasificar se siguieron por dos años.

PALABRAS CLAVE: Vasculitis cutánea. Vasculitis. Vasculitis leucocitoclástica. Púrpura. Lupus. Enfermedades reumáticas.

\begin{abstract}
Cutaneous vasculities (CV) represents a diagnostic challenge, occurs as primary cutaneous disorder or as a manifestation of other entities. Objective: To search the cause of CV. Methods: Patients with CV were prospectively evaluated. In all patients, skin biopsies were drawn, and direct immunofluorescence was done in most of the patients. American College of Rheumatology $(A C R)$ and Chapel Hill Consensus Conference Criteria (CHCC) were used for classification. Results: 32 patients were studied. There was female predominance (71.8\%). Children presented drug-associated CV or Schönlein-Henoch púrpura (SHP). Adults presented more frequently SHP, systemic lupus erythematosus or paraneoplastic vasculitis, other diagnosis as polyarteritis nodosa, microscopic polyangiitis, thrombotic vasculitis (post-puerperal), antiphospholipid syndrome, Churg-Strauss syndrome, and drug-associated CV were presented. Using the ACR and CHCC criteria, 50\% of cases were classified. Discussion: In our institution, during this work the etiologic diagnostic of CV increased more than twice. However, in the case
\end{abstract}

\author{
Correspondencia: \\ Lucía Rangel-Gamboa \\ Subdirección de Investigación \\ Hospital General Dr. Manuel Gea González \\ Calzada de Tlalpán 4800 \\ Col. Belisario Domínguez Sección XVI, Del. Tlalpan \\ C.P. 14080 , Ciudad de México, México \\ E-mail: draluciarangel@yahoo.com.mx \\ Fecha de recepción en versión modificada: 19-12-2016 \\ Fecha de aceptación: 30-12-2016 \\ DOI://dx.doi.org/10.24875/GMM.17002773
}

Gac Med Mex. 2018;154:62-67

Contents available at PubMed www.gacetamedicademexico.com 
of HSV or LA and SHP none of the proposed criteria had high specificity; other parameters were used to discern between both. Six patients remained as not classified. In our view, cryoglobulins and hepatitis serology do not seem useful unless patient's history supports they need to be done. Unclassified patients were followed-up closely for 2 years.

KEY WORDS: Cutaneous vasculities. Vasculitis. Purpura. Lupus. Rheumatic disease.

\section{Introducción}

La vasculitis es un proceso clinicopatológico caracterizado por inflamación y necrosis de los vasos sanguíneos, que conlleva su oclusión y produce isquemia tisular ${ }^{1,2}$. El diagnóstico etiológico de las VC constituye un reto, ya que pueden ser primarias o la manifestación inicial de enfermedades sistémicas diversas, como vasculitis necrotizante sistémica, enfermedades del tejido conectivo (LES), infecciones sistémicas o neoplasias ${ }^{3-5}$. La piel ofrece una oportunidad para el diagnóstico, al ser una fuente accesible para la realización de un estudio anatomopatológico ${ }^{6}$.

Las VC se clasifican en base a datos clínicos e histológicos, con los criterios del ACR, desde 19907, y con los criterios emitidos en la CHCC de 1992 (revisados en 2012) ${ }^{8}$; si bien estas clasificaciones no son del todo satisfactorias, continúan usándose en la práctica clínica ${ }^{9}$. Las vasculitis se agrupan de acuerdo al tamaño del vaso afectado en: a) vasculitis de grandes vasos, que incluye la arteritis de células gigantes y la arteritis de Takayasu' ${ }^{10}$; b) vasculitis de medianos vasos, como la PAN, la PAN cutánea y la enfermedad de Kawasaki ${ }^{11}$; y c) vasculitis de pequeños vasos, que pueden ser granulomatosas como la granulomatosis con poliangeítis, antes conocida como granulomatosis de Wegener ${ }^{12}$, y la eosinofilia con poliangeítis denominada también Síndrome de Churg-Strauss (SCS) ${ }^{13}$; y las no granulomatosas, que incluyen la $\mathrm{PAM}^{14}$, la $\mathrm{PSH}^{15}$, la vasculitis leucocitoclástica cutánea (VLC) y la crioglobulinemia esencial ${ }^{17}$.

Los factores etiológicos de las vasculitis son diversos e incluyen agentes infecciosos (15-20\%), enfermedades inflamatorias (15-20\%), medicamentos (10-15\%) y neoplasias (5\%), permaneciendo sin diagnóstico etiológico entre un 45 y un $55 \%$ de los $\operatorname{casos}^{5,18-20}$. En centros de referencia de tercer nivel, en pacientes adultos, las vasculitis asociadas a LES se presentan con mayor frecuencia ${ }^{21}$. El diagnóstico clínico de vasculitis debe confirmarse con estudio anatomopatológico, en el que se observan perdida de la arquitectura vascular, secundaria a la infiltración de sus paredes por neutrófilos, linfocitos o histiocitos; la presencia de necrosis fibrinoide es característica ${ }^{22}$. La inmunofluorescencia directa puede ser positiva en los vasos sanguíneos en etiologías específicas y resulta útil para clasificar y confirmar la presencia de vasculitis e identificar el tipo de inmunoglobulina (Ig) depositada en la pared vascular23; por ejemplo, la presencia de IgA se asocia a PSH, mientras que la IgM puede observarse en la vasculitis crioglobulinémica.

Las VC representan el $0.13 \%$ de la consulta en el Departamento de Dermatología del Hospital General Dr. Manuel Gea González. Sin embargo, no existía un protocolo de estudio para estos pacientes que se enfocara en determinar la etiología. En los años previos, la etiología se determinó en el $25 \%$ de los casos. Por tanto, el propósito de este trabajo fue determinar el diagnóstico etiológico de las VC en un hospital general durante un periodo de dos años y aumentar el porcentaje de diagnóstico etiológico. En este estudio se aplicaron las dos clasificaciones previamente mencionadas (tabla 1) $)^{24,25}$.

\section{Métodos}

Se incluyeron niños y adultos con diagnóstico clínico y anatomopatológico de VC que acudieron al Departamento de Dermatología del Hospital General Dr. Manuel Gea González durante un periodo de dos años. Para ser incluidos en el estudio los pacientes o sus representantes legales firmaron el consentimiento informado. El protocolo de estudio fue aprobado por el comité de ética e investigación de la institución y para la realización de la biopsia y toma de muestras se siguieron los principios de la declaración de Helsinki. Los pacientes se estudiaron de forma prospectiva, la evaluación incluyó una valoración clínica detallada y exploración física. Se tomaron dos muestras de las lesiones cutáneas por técnica de sacabocados de $4 \mathrm{~mm}$, una para tinción con hematoxilina y eosina y otra para inmunofluorescencia directa (DIF). Se solicitaron exámenes complementarios que incluían: biometría hemática, velocidad de sedimentación globular, química sanguínea, examen general de orina, sangre oculta en heces (guayaco), creatinina sérica, tiempos de coagulación, inmunoglobulinas totales (IgA, IgG, IgM), factor reumatoide, autoanticuerpos antinucleares (ANA), anticuerpos anticitoplasma de 
Gaceta Médica de México. 2018;154

Tabla 1. Nombres y definición de vasculitis adoptada por la CHCC y el ACR (modificado de Carlson, et al. 2005)

\begin{tabular}{|c|c|c|}
\hline & Chapel Hill Consensus Conference & American College of Rheumatology \\
\hline \multicolumn{3}{|l|}{ Vasculitis de grandes vasos } \\
\hline Arteritis de la temporal & $\begin{array}{l}\text { Arteritis granulomatosa de la aorta o sus ramas } \\
\text { principales (intracraneales) } \\
\text { Frecuentemente afecta la arteria temporal } \\
\text { Mayores de } 50 \text { años }\end{array}$ & $\begin{array}{l}50 \text { años } \\
\text { Cefalea } \\
\text { Arteria temporal anormal en el examen físico } \\
\text { Sedimentación globular elevada } \\
\text { La biopsia de la temporal muestra vasculitis }\end{array}$ \\
\hline Arteritis de Takayasu & $\begin{array}{l}\text { Inflamación granulomatosa de la aorta o sus } \\
\text { ramas principales } \\
\text { Frecuentemente en pacientes menores de } 50 \\
\text { años }\end{array}$ & $\begin{array}{l}\text { Menores de } 40 \text { años } \\
\text { Claudicación de miembros } \\
\text { Disminución de los pulsos arteriales } \\
\text { Diferencia en la presión arterial> } 10 \mathrm{mmHg} \text { entre los brazos } \\
\text { Arteriograma anormal }\end{array}$ \\
\hline \multicolumn{3}{|l|}{ Vasculitis de medianos vasos } \\
\hline Poliarteritis nodosa & $\begin{array}{l}\text { Inflamación necrotizante de medianas y } \\
\text { pequeñas arterias sin glomerulonefritis }\end{array}$ & $\begin{array}{l}\text { Pérdida de peso mayor a } 4 \text { kg } \\
\text { Livedo reticularis } \\
\text { Dolor testicular o hipersensibilidad } \\
\text { Mialgias, miopatía } \\
\text { Neuropatía } \\
\text { Hipertensión (diastólica > } 90 \text { mmHg) } \\
\text { Afección renal } \\
\text { Arteriografía anormal } \\
\text { En la biopsia se observa neutrófilos }\end{array}$ \\
\hline Enfermedad de Kawasaki & $\begin{array}{l}\text { Arteritis que afecta vasos grandes, medianos y } \\
\text { pequeños } \\
\text { Asociada con síndrome del nódulo linfático } \\
\text { mucocutáneo }\end{array}$ & \\
\hline \multicolumn{3}{|l|}{ Vasculitis de pequeños vasos } \\
\hline $\begin{array}{l}\text { Poliangeítis con } \\
\text { granulomatosis (granulomatosis } \\
\text { de Wegener) }\end{array}$ & $\begin{array}{l}\text { Lesiones granulomatosas pueden afectar el } \\
\text { tracto respiratorio, vasculitis granulomatosa que } \\
\text { afecta vasos de mediano y pequeño calibre }\end{array}$ & $\begin{array}{l}\text { Inflamación nasal u oral, presencia de nódulos, infiltrado } \\
\text { o cavitaciones en la Rx de tórax } \\
\text { Hematuria microscópica } \\
\text { Inflamación granulomatosa en la biopsia }\end{array}$ \\
\hline Síndrome de Churg-Strauss & $\begin{array}{l}\text { Inflamación granulomatosa rica en eosinófilos } \\
\text { que afecta al tracto respiratorio } \\
\text { Vasculitis necrotizante que afecta pequeños y } \\
\text { medianos vasos asociada con eosinofilia y asma }\end{array}$ & $\begin{array}{l}\text { Asma } \\
\text { Eosinófilos > 10\% } \\
\text { Neuropatía } \\
\text { Infiltrados pulmonares } \\
\text { Eosinófilos extravasculares en la biopsia }\end{array}$ \\
\hline Poliangeítis microscópica & $\begin{array}{l}\text { Vasculitis necrotizante con pocos o sin } \\
\text { depósitos inmunes que afecta los vasos } \\
\text { pequeños (capilares vénulas y arteriolas) } \\
\text { Glomerulonefritis } \\
\text { Afección pulmonar }\end{array}$ & \\
\hline Púrpura de Schönlein-Henoch & $\begin{array}{l}\text { Vasculitis con depósitos inmunes } \\
\text { predominantemente IgA que afecta vasos } \\
\text { pequeños (capilares, vénulas y arteriolas). } \\
\text { Frecuentemente afecta piel, intestino, glomérulo } \\
\text { y puede estar asociada a artralgias o artritis }\end{array}$ & $\begin{array}{l}\text { Púrpura palpable } \\
\text { Menores } 20 \text { años } \\
\text { Angina del intestino } \\
\text { En la biopsia se observan neutrófilos en la pared } \\
\text { vascular }\end{array}$ \\
\hline Vasculitis crioglobulinémica & $\begin{array}{l}\text { Vasculitis con depósitos inmunes por } \\
\text { crioglobulinas que afectan pequeños vasos y se } \\
\text { asocia con la presencia de crioglobulinas séricas } \\
\text { La piel y el glomérulo pueden afectarse }\end{array}$ & \\
\hline $\begin{array}{l}\text { Vasculitis leucocitoclástica } \\
\text { cutánea (vasculitis por } \\
\text { hipersensibilidad) }\end{array}$ & $\begin{array}{l}\text { Angeítis leucocitoclástica cutánea aislada } \\
\text { en ausencia de vasculitis sistémica o } \\
\text { glomerulonefritis }\end{array}$ & $\begin{array}{l}\text { Mayores } 16 \text { años } \\
\text { Asociada a medicamentos } \\
\text { Púrpura palpable } \\
\text { Erupción cutánea } \\
\text { Biopsia positiva }\end{array}$ \\
\hline
\end{tabular}

ACR: American College of Rheumatology; CHCC: Chapel Hill Consensus Conference. 
neutrófilo (ANCA), antimieloperoxidasa (MPO) y anti proteinasa 3 (PR-3), crioglobulinas, antifosfolípidos, $\lg \mathrm{G}$, IgA, IgM, complemento C3 y C4, así como serología para hepatitis $B$ y $C$. Se utilizaron los criterios del ACR y la CHCC para definir el diagnóstico etiológico. El diagnóstico operacional final se estableció por consenso entre los observadores al terminar el protocolo diagnóstico.

Cuarenta y cuatro pacientes con diagnóstico clínico de VC se incluyeron inicialmente en el protocolo, de los cuales doce se eliminaron: en ocho pacientes no se evidenció vasculitis en el estudio histopatológico, dos pacientes pediátricos fueron diagnosticados como púrpura trombocitopénica, otro paciente tuvo vasculitis sistémica de grandes vasos sin VC y, por último, un paciente no aceptó participar en el protocolo.

\section{Resultados}

De un total de cuarenta y cuatro pacientes con diagnóstico clínico de vasculitis, se incluyeron 5 niños y 27 adultos. Se observó predominio en el sexo femenino ( $71.8 \%$ vs. $28.2 \%)$. La media de edad fue de 37.75 años (rango de 6 a 73 años). Los niños $(n=5)$ tenían edades de 6, 8, 13 (dos pacientes) y 17 años. Las manifestaciones clínicas predominaron en miembros inferiores (69\%) y tronco, con morfología variada, siendo más frecuentes la púrpura palpable $(69 \%)$ y las máculas eritematopurpúricas (60\%) (Fig. 1). Algunos pacientes presentaron úlceras $(16 \%)$ y nudosida$\operatorname{des}(6 \%)$.

La PHS se diagnosticó en cuatro pacientes, tres adultos y un niño (13\%), la VC asociada a enfermedades de tejido conectivo se presentó en dos pacientes adultos, quienes fueron diagnosticados con LES $(6 \%)$, mientras que la vasculitis secundaria a ingesta de medicamentos o VHS se presentó en un adulto y dos niños (9\%), dos casos se asociaron a neoplasias, las cuales fueron diagnosticadas posteriormente a la presentación de la vasculitis. Se encontró un paciente con cada uno de los siguientes padecimientos: PAN, PAM, SAF, SCS y vasculitis trombóticas (pospuerperal). Utilizando los criterios del ACR para vasculitis se clasificaron el $44 \%$ de los pacientes y dos más usando los criterios ACR para LES, por tanto, un $50 \%$ de los pacientes fueron clasificados. El resto no satisfizo los criterios o no existían criterios para su enfermedad. Encontramos una sobreposición de los criterios para PSH y VHS en 12 casos, otros parámetros se usaron para discernir entre estos diagnósticos en los casos de duda. De estos, un 50\% tenía una $\mathrm{u}$ otra de las patologías, el resto fueron falsos positivos

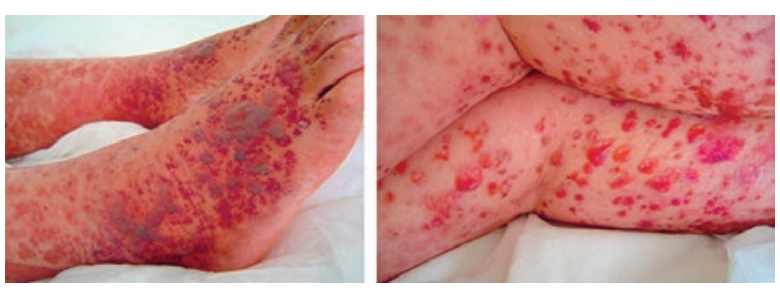

Figura 1. Purpura palpable en un paciente adulto con diagnóstico de púrpura de Scönlein-Henoch.

(cinco no pudieron clasificarse, otro fue diagnosticado durante el seguimiento como un síndrome paraneoplásico). Aplicando los criterios de la CHCC se clasificaron el $50 \%$ de los pacientes sin sobreposición de diagnóstico; de los doce pacientes con diagnóstico inicial de VLC, en tres de ellos se confirmó el diagnóstico y el resto fueron falsos positivos (una paraneoplasia, dos PSH y seis pacientes no pudieron clasificarse). Ningún paciente presento crioglobulinemia o serología positiva para hepatitis. Finalmente, 18 pacientes $(57 \%)$ se clasificaron con los criterios de la CHCC durante los dos años de seguimiento.

\section{Discusión}

En nuestra institución, se diagnóstica como VC 12.4 pacientes por año, lo que representa el $0.13 \%$ de la consulta, de acuerdo a la revisión realizada de los expedientes clínicos en un periodo de seis años. Previamente, la tasa de diagnóstico etiológico en los casos de VC era del $25 \%$. En este estudio, en el $57 \%$ de los casos con VC se llegó a un diagnostico etiológico, lo cual es similar a lo reportado en otros grupos $(51 \%)^{26}$. En la exploración inicial, el $56 \%$ de los pacientes no fueron clasificados, pero dos pacientes (que representan el 6\%) recibieron un diagnóstico etiológico durante el seguimiento que se realizó cada seis meses durante dos años, e incluyó exámenes de laboratorio y biopsia cuando fue necesario, lo cual facilitó obtener un diagnóstico oportuno y evitar complicaciones sistémicas. La VC fue más frecuente en las mujeres $(71.8 \%)$, con una edad media de presentación de 37.75 años.

En los niños el diagnóstico más frecuente fue la VHS, aun cuando en general se considera la PSH la causa más frecuente de vasculitis en niños; sin embargo, en esta serie de casos este hallazgo puede explicarse, ya que en el hospital los pacientes con PSH son referidos al servicio de pediatría, dado que los síntomas digestivos, renales y articulares suelen ser más severos que las lesiones cutáneas. En 
adultos, la causa más frecuente de VC fue la PSH, seguida de LES y las asociadas a neoplasias. Estudios previos reportaron una serie de 160 casos con VLC en la que el $10 \%$ de los casos se asoció con enfermedades del colágeno, similar a lo que reportamos en nuestros pacientes, mientras que otras series reportan hasta un $20 \%$. La VC se presentó como púrpura palpable en las extremidades inferiores en $22 / 32$ pacientes (68.7\%); en otros reportes, el $51 \%$ de los pacientes con VC presentan esta púrpura palpa$b^{27}{ }^{27}$. Las ulceras cutáneas como única manifestación se encontraron en el $15.6 \%$, incluyendo pacientes con los siguientes diagnósticos: PAN, SAF y LES, por lo que consideramos que los pacientes con úlceras en miembros inferiores que no responden al tratamiento convencional deben ser estudiados para eliminar la posibilidad de VC.

En los estudios de laboratorio, la IgA sérica estuvo elevada en 14 pacientes; dos con PSH, un caso de vasculitis por medicamentos, uno con LES y en 10 con otros diagnósticos. La PSH es una vasculitis sistémica en la cual la IgA tiene un papel fisiopatológico preponderante y puede encontrarse en las paredes vasculares en piel, riñones e intestino ${ }^{28,29}$. Sin embargo, la IgA sérica no siempre se encuentra elevada en estos pacientes (solo un $22 \%$ de los casos resultan positivos) y el ACR no la considera un criterio diagnóstic ${ }^{30}$. Por otra parte, la presencia de IgA en la pared vascular, detectada por DIF, es considerada un criterio diagnóstico de PSH para la CHCC.

En pacientes con diagnóstico de LES y VC, Ios ANA y el complemento se encontraron en límites normales, lo que se puede explicar porque los pacientes recibían tratamiento inmunosupresor para LES. Sin embargo, consideramos que es importante realizar estos exámenes durante el seguimiento, especialmente en pacientes con vasculitis linfocítica ${ }^{30}$. Los ANCA constituyen una prueba importante en el diagnóstico de poliangeítis con granulomatosis, PAM y SCS. La asociación de p-ANCA y MPO tiene un $99 \%$ de especificidad, de igual manera se asocian c-ANCA y PR-3 ${ }^{31-33}$. Blanco, et al., encontraron que un $30 \%$ de los pacientes adultos con VC presentaron una vasculitis sistémica o una enfermedad asociada (afección pulmonar, renal o mononeuritis); por tanto, es importante realizar estas pruebas en búsqueda de la etiología de una $\mathrm{VC}^{5}$. En relación a la DIF, se considera más apropiado realizar la biopsia en la lesión siempre que no se observe necrosis del tejido más que en la piel perilesional22.

La presencia de IgA en la pared vascular no es patognomónica, pero si altamente sensible (82\%) y especifica (73\%) para el diagnóstico de VLC y se asoció principalmente con $\mathrm{PSH}^{34,35}$. Recientemente se publicó una serie de ocho casos donde encontraron la presencia de $\lg A$ en vasculitis linfocíticas asociadas o no a LES ${ }^{36}$. La presencia de IgM o IgG sin IgA no elimina el diagnóstico de VLC, pero no lo apoya ${ }^{34}$. En nuestros casos observamos IgA en la pared vascular de cuatro pacientes con PSH, otros pacientes presentaron IgM y C3 o DIF negativa. Estos resultados pueden explicarse por la labilidad de la IgA, la cual desaparece rápidamente por proteólisis y fagocitosis en las lesiones necróticas. La presencia de IgA en los vasos cutáneos se asocia con daño renal pero no con la severidad del mismo ${ }^{37,38}$.

Es difícil realizar el diagnóstico de VC aplicando los criterios del ACR y de la CHCC, especialmente en pacientes con PSH o VHS, ya que las clasificaciones no ayudan a diferenciar entre estas dos entidades. La DIF con presencia de IgA en los vasos sanguíneos puede ayudar en algunos casos. Este problema se explica porque los criterios de la ACR se diseñaron con base en series de estudios clínicos, de laboratorio e histopatológicos que determinaron las características más sensibles y específicas para los diferentes tipos de vasculitis, pero no siempre ayudan a diferenciar entre dos posibilidades diagnósticas.

La evidencia en esta serie de casos indica que en la población mexicana, a diferencia de otras poblaciones, las crioglobulinas y la serología para hepatitis $B$ y $\mathrm{C}$ no son útiles como estudios iniciales, salvo que la historia clínica del paciente lo sugiera.

\section{Conclusiones}

Los criterios de la ACR no son en la actualidad completamente consistentes con las definiciones de las enfermedades específicas y no permiten siempre una aproximación diagnóstica precisa. Por otra parte, la CHCC de 1994 provee definiciones de las causas de vasculitis e incluye el uso de algunos marcadores cuando el estudio histológico no está disponible. Las definiciones de la CHCC fueron revisadas en $2012^{8}$, con objeto de incorporar nuevas terminologías, sin embargo, la CHCC entregó más una nomenclatura que una clasificación. En este contexto, consideramos que la inclusión de pruebas como los ANCA facilita el abordaje diagnóstico ${ }^{31,32,39}$. De acuerdo a nuestros hallazgos, proponemos el protocolo de estudio expuesto en la figura 2 para los pacientes con vasculitis. 


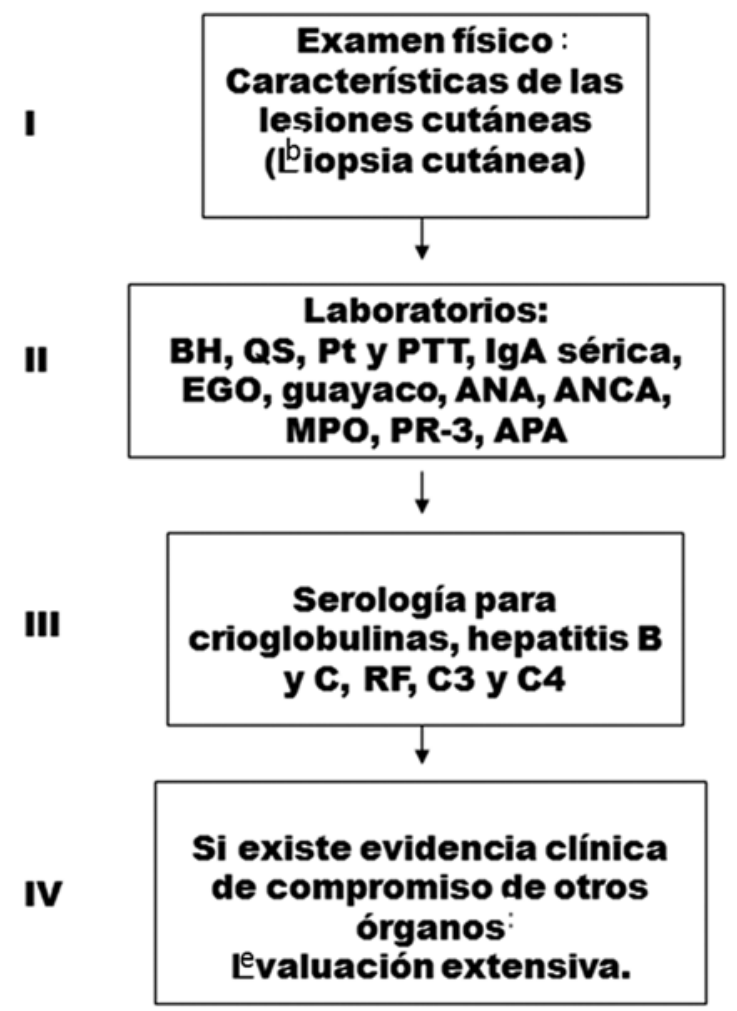

Figura 2. Flujograma. ANA: autoanticuerpos antinucleares; ANCA: anticuerpos anticitoplasma de neutrófilo; APA: anticuerpos antifosfolípidos (por sus siglas en inglés); $B H$ : biometría hemática; EGO: examen general de orina; MPO: antimieloperoxidasa; PR-3: proteinasa 3; Pt: tiempo de protrombina; PTT: tiempo parcial de tromboplastina; QS: química sanguínea.

\section{Bibliografía}

1. Gonzalez-Gay MA, Garcia-Porrua C, Pujol RM. Clinical approach to cutaneous vasculitis. Curr Opin Rheumatol. 2005;17(1):56-61.

2. Sneller M, Fauci A. Pathogenesis of vasculitis sindromes. Med Clin North Am. 1997;81(1):221-42.

3. Ramirez GA, Weyand C, Vaglio A, et al. Editorial: Vascular inflammation in systemic autoimmunity. Front Immunol. 2016;21(7):471.

4. Chen KR, Carlson JA. Clinical approach to cutaneous vasculitis. Am J Clin Dermatol. 2008;9(2):71-92.

5. Blanco R, Martínez-Taboada V, Rodríguez-Valverde V. Cutaneous vasculitis in children and adults: associated diseases and etiologic factors in 303 patients. Medicine. 1998;77(6):403-18.

6. Crowson AN, Minm M, Magro C. Cutaneous vasculitis: a Review. J Cutan Pathol. 2003:30(3):161-73.

7. Bloch DA, Michel BA, Hunter GG, et al. The American College of Rheumatology. Development of classification and response criteria for the classification of vasculitis, patients and methods. Arthritis Rheum. 1990;2990(33):1068-73.

8. Jennette JC, Falk RJ, Bacon PA, et al. 2012 revised international Chapel Hill consensus conference nomenclature of vasculitides. Arthris Rheuma. 2013;65:1-11.

9. Luqmani RA, Suppiah R, Grayson PC, et al. Nomenclature and classification of vasculitis - update on the ACR/EULAR diagnosis and classification of vasculitis study (DCVAS). Clin Exp Immunol. 2011;164(suppl1):11-3.
10. Töpel I, Zorger N, Steinbauer M. Inflammatory diseases of the aorta. Gefässchirurgie 2016;21(Suppl 2):S80-6.

11. Dillon MJ, Eleftheriou D, Brogan P. Medium-size-vessel vasculitis. Pediatr Nephrol 2010;25(9):1641-52.

12. Wojciechowska J, Krajewski W, Krajewski P, et al. Granulomatosis with polyangiitis in otolaryngology. Clin Experimen Otor. 2016;9(1):8-13.

13. Benallegue $N$, Lozach $P$, Belizna $C$, et al. Acute coronary vasospasm in a patient with eosinophilic granulomatosis with polyangitis following NSAID administration: A case report. Medicine (Baltimore). 2016;95(47):e5259.

14. García-Nava M, Mateos-Toledo $H$, Guevara-Canseco AP, et al. Early interstitial lung disease in microscopic polyangiitis: case report and literature review. Reumatol Clin. 2016;2:S1699-258X(16)30132-2.

15. Hong J, Yang HR, Laboratory markers indicating gastrointestinal involvement of Henoch-Schönlein purpura in children. Pediatr Gastroenterol Hepatol Nutr. 2015;18(1):39-47.

16. Monjazeb S, Philips RC, Wilkerson M. A case of leukocytoclastic vasculitis following influenza vaccination. JAAD Case Rep. 2016;17(4):340-2.

17. Ozen S, Ruperto N, Dillon MJ, et al. EULAR/PReS endorsed consensus criteria for the classification of childhood vasculitides. Ann Rheum Dis. 2006;65:936-41.

18. Diplomatico M, Gicchino MF, Ametrano $O$, et al. A case of urticarial vasculitis in a female patient with lupus: Mycoplasma pneumoniae infection or lupus reactivation? Rheumatol Int. 2017;37(5):837-40.

19. Sáenz de Santa María M, Morales-Cabeza C, Noguerado-Mellado B, et al. Cutaneous leukocytoclastic vasculitis due to amoxicillin hypersensitivity. Ann Allergy Asthma Immunol. 2016;117(4):446-7.

20. Lee SH, Kim JH, Park S, et al. Pulmonary leukocytoclastic vasculitis as an initial presentation of myelodysplastic syndrome. Tuberc Respir Dis. 2016;79(4):302-6.

21. Ruelas Villavicencio AL, García Hidalho L, Reyes Gutiérrez E, et al. Causas de vasculitis cutánea en 59 pacientes mexicanos. Experiencia de un centro de tercer nivel. Dermatología Rev Mex. 2011;55(6):334-1.

22. Carlson A. The histological assessment of cutaneous vasculitis. Histopathology. 2010;56:3-23.

23. Pulido-Pérez A, Avilés-Izquierdo JA Suárez-Fernández R. Vasculitis cutáneas. Actas Dermosiiliogr. 2012;103(3):179-91.

24. Carlson A, Ng BT, Chen KR. Cutaneous vasculitis update: Diagnostic criteria classification, epidemiology, etiology, pathogenesis, evaluation and prognosis. Am J Dermatopathol. 2005;27:504-28.

25. Fiorentino D. Cutaneous vasculitis. J Am Acad Dermatol. 2003; 48(3):311-40.

26. Al-Mutairi N. Spectrum of cutaneous vasculitis in adult patients from the Fawiniya region of Kuwait. Med Princ Pract. 2008;17(1):43-8.

27. Gibson L. Cutaneous vasculitis update. Dermatol Clin. 2001;19(4):603-15.

28. Kawakami T, Yamazaki M, Mizoguchi M, et al. High titer of serum antiphospholipid antibody levels in adult Henoch-Schönlein purpura and cutaneous leukocytoclastic angiitis. Arthritis Rheum. 2008;59(4):561-7.

29. Davin JC, Weening J. Diagnosis of Henoch-Schönlein purpura: renal or skin biopsy? Ped Nephrol. 2003;1201-3.

30. Oh C, Lee SH, Heo CP. A case suggesting lymphocytic vasculitis as a presenting sign of early undifferenciated connective tissue disease. Am J Dermatopathol. 2003;25(5):423-7.

31. Monach PA. Biomarkers in Vasculitis. Curr Opin Rheumatol. 2014; 26(1):24-30

32. Wiik A. Rational use of ANCA in the diagnosis of vasculitis. Rheumatol. 2002:41:481-3.

33. Mohan N, Kerr G. Diagnosis of vasculitis. Best Pact Research Clin Rheumatol. 2001;15(2):203-23.

34. Barnadas M, Perez E, Gich I. Diagnostic, prognostic and pathogenic value of the direct immunofluorescence test in cutaneous leucocytoclastic vasculitis. Int J Dermatol. 2004;43:19-26.

35. Fervenza F. Henoch-Schönlein purpura nephritis. Int J Dermatol. 2003;42:170-7.

36. Crowson A, Magro C, Usmani A, et al. Immunoglobulin A-associated lymphocytic vasculopathy: a clinicopathologic study of eight patients. J Cutan Pathol. 2002;29:596-601.

37. Sano $H$, Izumida $M$, Shimizu $H$. Risk factors of renal involvement and significant proteinuria in Henoch-Schönlein purpura. Eur $\mathrm{J}$ Pediatr. 2002:196-201.

38. Davin JC, Weening J. Henoch-Schönlein purpura: an update. Eur J Pediatr. 2001:689-95.

39. Craven A, Robson J, Ponte C, et al. ACR/EULAR-endorsed study to develop Diagnostic and Classification Criteria for Vasculitis (DCVAS). Clin Exp Nephrol. 2013;17:619-21. 